\title{
Multiple periodic solutions for the second-order nonlinear difference equations
}

\author{
Sheng $\mathrm{Ma}^{1}$, Zhihua $\mathrm{Hu}^{1}$ and Qin Jiang ${ }^{1 *}$
}

\author{
"Correspondence: \\ jiangqin999@126.com \\ ${ }^{1}$ College of Mathematics and \\ Physics, Huanggang Normal \\ University, Hubei, People's Republic \\ of China
}

\begin{abstract}
Multiple periodic solutions for the equation

$$
\Delta\left(p_{n}\left(\Delta x_{n-1}\right)^{\delta}\right)+a_{n} x_{n}^{\delta}=\nabla F\left(n, x_{n}\right), \quad n \in \mathbb{Z},
$$

are obtained via variational method and saddle-point theorem of Brezis and Nirenberg. Our main results extend some earlier results. An example is given.
\end{abstract}

MSC: 58E50; 39A $11 ; 39 \mathrm{~A} 10$

Keywords: Multiple solutions; Difference equation; PS condition; Second-order; Saddle-point theorem

\section{Introduction}

Let $\mathbb{Z}, \mathbb{N}$. And $\mathbb{R}$ be the sets of all integers, all positive integers, and all real numbers respectively. Set $\mathbb{Z}[a, b]=\{a, a+1, \ldots, b\}$ for $a \leq b, a, b \in \mathbb{Z}$.

We consider the existence of multiple solutions for the following difference equation:

$$
\Delta\left(p_{n}\left(\Delta x_{n-1}\right)^{\delta}\right)+q_{n} x_{n}^{\delta}=\nabla F\left(n, x_{n}\right), \quad n \in \mathbb{Z} .
$$

Here $\Delta x_{n}=x_{n+1}-x_{n}, \Delta^{2} x_{n}=\Delta\left(\Delta x_{n}\right)$, the odd integer $\delta>0$ is the ratio, real sequences $\left\{p_{n}\right\},\left\{q_{n}\right\}$ and the function $F(n, x)=\int_{0}^{x} \nabla F(n, s) d s$ satisfy

$$
p_{n}=p_{n+T}>0, \quad q_{n}=q_{n+T}, \quad F(n+T, x)=F(n, x), \quad n \in \mathbb{Z},
$$

for a fixed $T \in \mathbb{Z}$.

Difference equations are widely found in mathematics itself and in its applications to combinatorial analysis, quantum physics, chemical reactions, and so on. Many authors were interested in difference equations and obtained many significant conclusions; see, for instance, the papers $[1-3,5-20]$. Various methods have been used to deal with the existence of solutions to discrete problems, we refer to the fixed point theorems in cones in [12] and the variational method in $[2,3,5-11,13,14,18-20]$. In 2003, in [10, 11] Yu and Guo made a new variational structure to handle discrete equations and obtained good conclusions on the solvability condition of a periodic solution. This new variational structure represents an important advance as it allows us to prove multiplicity results as well.

(c) The Author(s) 2018. This article is distributed under the terms of the Creative Commons Attribution 4.0 International License (http://creativecommons.org/licenses/by/4.0/), which permits unrestricted use, distribution, and reproduction in any medium, provided you give appropriate credit to the original author(s) and the source, provide a link to the Creative Commons license, and indicate if changes were made. 
When $\delta=1$, equation (1) has been considered in [17] and [19]. When $\delta \neq 1$ and $\nabla F(n, x)=0$, equation (1) has been also studied. For example, in [1] and [8], the authors were interested in the results on disconjugacy, disfocality, oscillation, and the asymptotic behavior. In [20] the authors were interested in the results on the existence of positive solutions. However, to the best of our knowledge, when $\delta \neq 1$ and $\nabla F(n, x) \neq 0$, besides [6] and [18], in the literature there are no results on the solvability condition of periodic solutions for equation (1). By employing the mountain pass lemma, in [6] the authors proved that there are at least two nontrivial periodic solutions of equation (1) under the following conditions:

(a) $q_{n}<0, \forall n \in \mathbb{Z}[1, T]$.

(b) For each $n \in \mathbb{Z}, \lim _{x \rightarrow 0} \frac{\nabla F(n, x)}{|x|^{\delta}}=0$.

(c) There exist constants $a_{1}>0, a_{2}>0$, and $\beta>\delta+1$ such that

$$
F(n, x) \leq-a_{1}|x|^{\beta}+a_{2}, \quad \forall x \in \mathbb{R}
$$

To get the critical points, condition (c) is essential, especially, $\beta>\delta+1$. Later, by virtue of the saddle-point theorem, in [18] the authors obtained the periodic solution of equation (1) under condition (a) and the following assumptions:

(d) There are constants $R_{1}>0$ and $\alpha \in(1,2)$ such that

$$
\frac{\alpha}{2}(\delta+1) F(n, x) \leq x \nabla F(n, x)<0 \quad \text { for } n \in \mathbb{Z} \text { and }|x| \geq R_{1} \text {. }
$$

(e) There are constants $a_{3}>0, a_{4}>0$, and $\gamma \in(1, \alpha]$ such that

$$
F(n, x) \leq-a_{3}|x|^{\frac{\gamma}{2}(\delta+1)}+a_{4}, \quad \forall x \in \mathbb{R}
$$

From condition (e) we have $\beta=\frac{\gamma}{2}(\delta+1)<\delta+1$.

Hence it is natural for us to consider the case $\beta=\delta+1$. In the present papere, the motivation comes from the recent papers $[6,7,14,18]$. By virtue of the minimax methods with variational techniques, the solvability conditions on multiple periodic solutions are proved for difference equation when $\beta=\delta+1$. In particular, our results complement and generalize the results in [6] and [18].

\section{Preliminaries}

To obtain multiple periodic solutions via variational techniques, we state the corresponding variational structure for equation (1). Let $S$ be the sequence

$$
S=\left\{u_{n}\right\}=\left(\ldots, u_{-n}, \ldots, u_{-1}, u_{0}, u_{1}, \ldots, u_{n}, \ldots\right)=\left\{u_{n}\right\}_{-\infty}^{+\infty}
$$

Define

$$
a u+b v=\left\{a u_{n}+b v_{n}\right\}, \quad \forall u, v \in S, a, b \in \mathbb{R} .
$$

This yields that $S$ is a vector space. For any fixed $T \in \mathbb{N}$, let $E_{T}$ be the subspace of $S$ defined as

$$
E_{T}=\left\{u=\left\{u_{n}\right\} \in S \mid u_{n+T}=u_{n}, n \in \mathbb{Z}\right\}
$$


Define the inner product $\langle\cdot, \cdot\rangle_{E_{T}}$ and norm $\|\cdot\|_{E_{T}}$ in $E_{T}$ as follows:

$$
\langle u, v\rangle_{E_{T}}=\sum_{i=1}^{T} u_{i} v_{i} \text { and }\|u\|_{E_{T}}=\|u\|=\left(\sum_{j=1}^{T} u_{j}^{2}\right)^{\frac{1}{2}} \quad \forall u, v \in E_{T} .
$$

Obviously, the space $E_{T}$ is finite dimensional. Set

$$
\|u\|_{\delta+1}=\left(\sum_{j=1}^{T} u_{j}^{\delta+1}\right)^{\frac{1}{\delta+1}}
$$

Since $\delta+1 \geq 2$, by the Hölder inequality we have

$$
\|u\| \leq T^{\frac{\delta-1}{\delta+1}}\|u\|_{\delta+1} \leq T^{\frac{1}{2}}\|u\|_{\delta+1}, \quad u \in E_{T} .
$$

On the other hand, we have

$$
\left(\sum_{j=1}^{T} u_{j}^{\delta+1}\right)^{\frac{2}{\delta+1}} \leq \sum_{j=1}^{T} u_{j}^{2}, \quad u \in E_{T}
$$

Obviously,

$$
T^{-\frac{1}{2}}\|u\| \leq\|u\|_{\delta+1} \leq\|u\|
$$

for all $u \in E_{T}$. Let

$$
\varphi(x)=-\frac{1}{\delta+1} \sum_{n=1}^{T} p_{n}\left(\Delta x_{n-1}\right)^{\delta+1}+\frac{1}{\delta+1} \sum_{n=1}^{T} q_{n} x_{n}^{\delta+1}-\sum_{n=1}^{T} F\left(n, x_{n}\right), \quad \forall x \in E_{T} .
$$

Then $\varphi \in C^{1}\left(E_{T}, \mathbb{R}\right)$, and we have the partial derivative

$$
\frac{\partial \varphi}{\partial x_{n}}=\Delta\left(p_{n}\left(\Delta x_{n-1}\right)^{\delta}\right)+q_{n} x_{n}^{\delta}-\nabla F\left(n, x_{n}\right), \quad n \in \mathbb{Z}[1, T]
$$

via $x_{0}=x_{T}, x_{1}=x_{T+1}$. Thus $x \in E_{T}$ satisfies $\varphi^{\prime}(x)=0$ if and only if $x$ satisfies equation (1) for any $n \in \mathbb{Z}[1, T]$. To seek the $T$-periodic solutions for equation (1) is to find the critical points of functional $\varphi$ since $x_{n}=x_{T+n}$ and $\nabla F(n+T, x)=\nabla F(n, x)$.

Set the $T \times T$ matrix

$$
P=\left[\begin{array}{cccccc}
2 & -1 & 0 & \ldots & 0 & -1 \\
-1 & 2 & -1 & \ldots & 0 & 0 \\
0 & -1 & 2 & \ldots & 0 & 0 \\
\cdots & \ldots & \ldots & \ldots & \ldots & \ldots \\
0 & 0 & 0 & \ldots & 2 & -1 \\
-1 & 0 & 0 & \ldots & -1 & 2
\end{array}\right] .
$$

From the matrix theory we know that

$$
\lambda_{k}=2\left(1-\cos \frac{2 k \pi}{T}\right), \quad k=1,2, \ldots, T-1
$$


are the eigenvalues of $P$ and $\lambda_{0}=0, \lambda_{1}>0, \lambda_{2}>0, \ldots, \lambda_{T-1}>0$. Thus we have

$$
\lambda_{\min }=\min \left\{\lambda_{1}, \lambda_{2}, \ldots, \lambda_{T-1}\right\}=\lambda_{1}=2\left(1-\cos \frac{2 \pi}{T}\right) .
$$

Let

$$
W=\operatorname{ker} P=\left\{x \in E_{T} \mid P x=0\right\} .
$$

Then

$$
W=\left\{x \in E_{T} \mid x=\{c\}, c \in \mathbb{R}\right\}
$$

Set $V=W^{\perp}$. Clearly, $E_{T}=V \oplus W$. For convenience, we identify $x \in E_{T}$ with $x=$ $\left(x_{1}, x_{2}, \ldots, x_{T}\right)^{T}$.

\section{Main results and proofs}

Set

$$
p_{\min }=\min _{n \in \mathbb{Z}[1, T]} p_{n}>0, \quad q_{\min }=\min _{n \in \mathbb{Z}[1, T]} q_{n}, \quad q_{\max }=\max _{n \in \mathbb{Z}[1, T]} q_{n} .
$$

We study equation (1) under the following conditions:

(F1) $q_{\max }-q_{\min }<p_{\min } \lambda_{1}^{\frac{\delta+1}{2}} \frac{1}{T^{\delta+1}}$.

(F2) There exist constants $\rho_{0}>0$ and

$$
a_{1} \in\left(\left(\frac{q_{\max }-q_{\min }}{p_{\min } \lambda_{1}^{\frac{\delta+1}{2}}}\right)^{\frac{1}{\delta+1}}, \frac{1}{T}\right)
$$

such that

$$
\frac{q_{\max }-p_{\min } \lambda_{1}^{\frac{\delta+1}{2}} a_{1}^{\delta+1}}{\delta+1}|x|^{\delta+1} \leq F(n, x) \leq \frac{q_{\min }}{\delta+1}|x|^{\delta+1}
$$

for all $n \in \mathbb{Z}$ and $|x| \leq \rho_{0}$.

(F3) $\lim \sup _{|x| \rightarrow \infty} \frac{F(n, x)}{|x|^{\delta+1}}<-\frac{A}{\delta+1}$, where $A=\max _{n \in \mathbb{Z}[1, T]}\left[2^{\delta+1}\left(p_{n}+p_{n+1}\right)-q_{n}\right]$.

(F4) There are constants $\rho_{1}>0$ and $a_{2} \in\left(0, \frac{1}{T}\right)$ such that

$$
-\frac{p_{\min } \lambda_{1}^{\frac{\delta+1}{2}} a_{2}^{\delta+1}}{\delta+1}|x|^{\delta+1} \leq F(n, x) \leq 0
$$

for all $n \in \mathbb{Z}$ and $|x| \leq \rho_{1}$.

(F5) $\lim \sup _{|x| \rightarrow \infty} \frac{F(n, x)}{|x|^{\delta+1}}<-\frac{B}{\delta+1}$, where $B=\max _{n \in \mathbb{Z}[1, T]}\left[2^{\delta+1}\left(p_{n}+p_{n+1}\right)\right]$.

Now we state our main results.

Theorem 3.1 Under hypotheses (F1), (F2), and (F3), equation (1) has at least two nontrivial T-periodic solutions.

For the particular case $q_{n}=0$ in Theorem 3.1, we easily obtain the following result. 
Corollary 3.2 Under hypotheses (F4) and (F5), equation (1) has at least two nontrivial T-periodic solutions.

Remark 3.3 Theorem 3.1 generalizes Theorem 3.2 in [6]. On the one hand, the sequence $\left\{q_{n}\right\}$ can be sign-changing. However, $\left\{q_{n}\right\}$ is negative in Theorem 3.2 of [6]. On the other hand, conditions (F2) and (F3) in our Theorem 3.1 are weaker than the corresponding conditions in Theorem 3.2. There are functions $F$ satisfying Theorem 3.1 but not fulfilling Theorem 3.2 in [6] and Theorem 1.3 in [18]. For example, set

$$
F(n, x)=-|x|^{\delta+1} \ln (1+|x|)-\frac{1}{2(\delta+1)} \lambda_{1}^{\frac{\delta+1}{2}}\left(\frac{1}{2 T}\right)^{\delta+1}|x|^{\delta+1}
$$

and

$$
q_{n}=\frac{1}{4} \lambda_{1}^{\frac{\delta+1}{2}}\left(\frac{1}{2 T}\right)^{\delta+1} \sin \frac{2 \pi n}{T}, \quad p_{n}=2+\sin \frac{2 \pi n}{T} .
$$

Clearly, $\left\{q_{n}\right\}$ and $\left\{p_{n}\right\}$ are $T$-periodic, $p_{\min }=1>0$, and

$$
\frac{q_{\max }-q_{\min }}{p_{\min } \lambda_{1}^{\frac{\delta+1}{2}}}=\frac{1}{2}\left(\frac{1}{2 T}\right)^{\delta+1}<\left(\frac{1}{T}\right)^{\delta+1},
$$

which implies that $\left\{q_{n}\right\}$ and $\left\{p_{n}\right\}$ satisfy (F1) in Theorem 3.1 but do not satisfy the corresponding condition of Theorem 3.2 of [6]. A simple computation yields

$$
\lim _{|x| \rightarrow 0} \frac{F(n, x)}{|x|^{\delta+1}}=-\frac{1}{2(\delta+1)} \lambda_{1}^{\frac{\delta+1}{2}}\left(\frac{1}{2 T}\right)^{\delta+1} .
$$

Put $a_{1}=\frac{1}{2 T}$. Clearly,

$$
\frac{q_{\max }-q_{\min }}{p_{\min } \lambda_{1}^{\frac{\delta+1}{2}}}<a_{1}^{\delta+1}<\left(\frac{1}{T}\right)^{\delta+1}
$$

and

$$
\frac{q_{\max }-p_{\min } \lambda_{1}^{\frac{\delta+1}{2}} a_{1}^{\delta+1}}{\delta+1}<-\frac{1}{2(\delta+1)} \lambda_{1}^{\frac{\delta+1}{2}}\left(\frac{1}{2 T}\right)^{\delta+1}<\frac{q_{\min }}{\delta+1} .
$$

So $F$ satisfies (F2). Further, we have

$$
\lim _{|x| \rightarrow \infty} \sup \frac{F(n, x)}{|x|^{\delta+1}}=-\infty,
$$

which implies that such a function $F$ satisfies condition (F3) of Theorem 3.1 but does not satisfy the corresponding condition of Theorem 3.2 in [6] and the corresponding condition of Theorem 1.3 in [18]. Moreover, our conclusion complements the results of Theorem 3.2 in [6] and Theorem 1.3 in [18].

The proof of Theorem 3.1 uses the following saddle-point theorem of Brezis and Nirenberg. 
Lemma 3.4 ([4]) Let $X$ be a Banach space with $X=X_{1}+X_{2}$, where $\operatorname{dim} X_{2}<\infty$, and let $\varphi$ be a $C^{1}$ function on $X$ with $\varphi(0)=0$ satisfying the PS condition. Suppose that, for some $\delta_{0}>0$,

$\left(I_{1}\right) \varphi(v) \geq 0$, for $v \in X_{1}$ with $\|v\| \leq \delta_{0}$, and

$\left(I_{2}\right) \varphi(v) \leq 0$, for $v \in X_{2}$ with $\|v\| \leq \delta_{0}$.

If $\varphi$ is bounded from below and $\inf _{X} \varphi<0$, then $\varphi$ has at least two nonzero critical points.

Proof of Theorem 3.1 Set

$$
W=X_{1}, \quad V=X_{2} .
$$

First, we claim that $\varphi$ satisfies the PS condition. Now let $\left\{x^{(k)}\right\}$ be a sequence for $\varphi$ such that $\left\{\varphi\left(x^{(k)}\right)\right\}$ is bounded and $\varphi^{\prime}\left(x^{(k)}\right) \rightarrow 0$ as $k \rightarrow \infty$. Then we need to verify that there is a convergent subsequence of $\left\{x^{(k)}\right\}$. Since $E_{T}$ is finite dimensional, we only need to prove that $\left\{x^{(k)}\right\}$ is bounded.

By (F3) there exist constants $b_{1}>\frac{A}{\delta+1}, b_{2}>0$ satisfying

$$
F(n, x) \leq-b_{1}|x|^{\delta+1}+b_{2}
$$

for all $n \in \mathbb{Z}[1, T]$ and $x \in \mathbb{R}$. In view of (2) and (3), for any $k \in \mathbb{N}$, we obtain

$$
\begin{aligned}
\varphi\left(x^{(k)}\right)= & -\frac{1}{\delta+1} \sum_{n=1}^{T}\left[p_{n}\left(x_{n}^{(k)}-x_{n-1}^{(k)}\right)^{\delta+1}-q_{n}\left(x_{n}^{(k)}\right)^{\delta+1}\right]-\sum_{n=1}^{T} F\left(n, x_{n}^{(k)}\right) \\
\geq & -\frac{1}{\delta+1} \sum_{n=1}^{T} p_{n} 2^{\delta+1}\left(\left(x_{n}^{(k)}\right)^{\delta+1}+\left(x_{n-1}^{(k)}\right)^{\delta+1}\right)+\frac{1}{\delta+1} \sum_{n=1}^{T} q_{n}\left(x_{n}^{(k)}\right)^{\delta+1} \\
& -\sum_{n=1}^{T} F\left(n, x_{n}^{(k)}\right) \\
\geq & -\frac{2^{\delta+1}}{\delta+1} \sum_{n=1}^{T}\left(p_{n}+p_{n+1}\right)\left(x_{n}^{(k)}\right)^{\delta+1}+\frac{1}{\delta+1} \sum_{n=1}^{T} q_{n}\left(x_{n}^{(k)}\right)^{\delta+1}-\sum_{n=1}^{T} F\left(n, x_{n}^{(k)}\right) \\
\geq & -\frac{1}{\delta+1} \sum_{n=1}^{T}\left[2^{\delta+1}\left(p_{n}+p_{n+1}\right)-q_{n}\right]\left(x_{n}^{(k)}\right)^{\delta+1}+b_{1} \sum_{n=1}^{T}\left(x_{n}^{(k)}\right)^{\delta+1}-b_{2} T \\
\geq & -\frac{A}{\delta+1}\left\|x^{(k)}\right\|_{\delta+1}^{\delta+1}+b_{1}\left\|x^{(k)}\right\|_{\delta+1}^{\delta+1}-b_{2} T \\
\geq & \left(b_{1}-\frac{A}{\delta+1}\right) T^{-\frac{\delta+1}{2}}\left\|x^{(k)}\right\|^{\delta+1}-b_{2} T .
\end{aligned}
$$

Since $\left\{\varphi\left(x^{(k)}\right)\right\}$ is bounded and $b_{1}-\frac{A}{\delta+1}>0,\left\{x^{(k)}\right\}$ is bounded in $E_{T}$. In the finitedimensional space $E_{T}$, such a bounded consequence $\left\{x^{(k)}\right\}$ has a convergent subsequence. Hence $\varphi$ satisfies the PS condition.

Clearly, $\varphi(0)=0$ by (F2). Hence $x=0$ is a trivial solution of equation (1).

On the one hand, for any $w \in W$ with $\|w\| \leq \rho$ and $n \in \mathbb{Z}[1, T]$, due to (F2), we obtain

$$
\varphi(w)=\frac{1}{\delta+1} \sum_{n=1}^{T} q_{n} w^{\delta+1}-\sum_{n=1}^{T} F(n, w) \geq \frac{1}{\delta+1} \sum_{n=1}^{T} q_{n} w^{\delta+1}-\frac{1}{\delta+1} \sum_{n=1}^{T} q_{n} w^{\delta+1}=0 .
$$

So condition $\left(I_{1}\right)$ of Lemma 3.4 is verified. 
For all $x \in E_{T}$, by the Hölder inequality we have

$$
\sum_{n=1}^{T}\left(\Delta x_{n-1}\right)^{2} \leq\left(\sum_{n=1}^{T} 1^{\frac{\delta+1}{\delta-1}}\right)^{\frac{\delta-1}{\delta+1}} \cdot\left(\sum_{n=1}^{T}\left(\Delta x_{n-1}\right)^{\delta+1}\right)^{\frac{2}{\delta+1}}=T^{\frac{\delta-1}{\delta+1}} \cdot\left(\sum_{n=1}^{T}\left(\Delta x_{n-1}\right)^{\delta+1}\right)^{\frac{2}{\delta+1}}
$$

Then a simple computation yields

$$
\left[\sum_{n=1}^{T}\left(\Delta x_{n-1}\right)^{2}\right]^{\frac{\delta+1}{2}} \leq T^{\frac{\delta-1}{2}} \sum_{n=1}^{T}\left(\Delta x_{n-1}\right)^{\delta+1} \leq T^{\frac{\delta+1}{2}} \sum_{n=1}^{T}\left(\Delta x_{n-1}\right)^{\delta+1}
$$

On the other hand, by (F2) and (5), for all $n \in \mathbb{Z}[1, T]$ and $x \in V=W^{\perp}$ with $\|x\| \leq \rho$, we have

$$
\begin{aligned}
\varphi(x) & \leq-\frac{1}{\delta+1} \sum_{n=1}^{T} p_{n}\left(\Delta x_{n-1}\right)^{\delta+1}+\frac{1}{\delta+1} \sum_{n=1}^{T} q_{n} x_{n}^{\delta+1}-\frac{q_{\max }-p_{\min } \lambda_{1}^{\frac{\delta+1}{2}} a_{1}^{\delta+1}}{\delta+1} \sum_{n=1}^{T} x_{n}^{\delta+1} \\
& \leq-\frac{p_{\min }}{\delta+1} \sum_{n=1}^{T}\left(\Delta x_{n-1}\right)^{\delta+1}+\frac{q_{\max }}{\delta+1} \sum_{n=1}^{T} x_{n}^{\delta+1}-\frac{q_{\max }-p_{\min } \lambda_{1}^{\frac{\delta+1}{2}} a_{1}^{\delta+1}}{\delta+1} \sum_{n=1}^{T} x_{n}^{\delta+1} \\
& \leq-\frac{p_{\min }}{\delta+1}\left(\frac{1}{T}\right)^{\frac{\delta+1}{2}}\left[\sum_{n=1}^{T}\left(\Delta x_{n-1}\right)^{2}\right]^{\frac{\delta+1}{2}}+\frac{p_{\min } \lambda_{1}^{\frac{\delta+1}{2}} a_{1}^{\delta+1}}{\delta+1} \sum_{n=1}^{T} x_{n}^{\delta+1} \\
& \leq-\frac{p_{\min }}{\delta+1} \lambda_{1}^{\frac{\delta+1}{2}}\left(\frac{1}{T}\right)^{\frac{\delta+1}{2}}\|x\|^{\delta+1}+\frac{p_{\min } \lambda_{1}^{\frac{\delta+1}{2}} a_{1}^{\delta+1}}{\delta+1} T^{\frac{\delta+1}{2}}\|x\|^{\delta+1} \\
& \leq\left(\left(a_{1} \sqrt{T}\right)^{\delta+1}-\left(\frac{1}{\sqrt{T}}\right)^{\delta+1}\right) \frac{p_{\min }}{\delta+1} \lambda_{1}^{\frac{\delta+1}{2}}\|x\|^{\delta+1} .
\end{aligned}
$$

Since $a_{1}<\frac{1}{T}$, we have $a_{1} \sqrt{T}<\frac{1}{\sqrt{T}}$. Then

$$
\left(a_{1} \sqrt{T}\right)^{\delta+1}-\left(\frac{1}{\sqrt{T}}\right)^{\delta+1}<0
$$

Thus by (6), (7), and $p_{\min }>0$, we conclude that

$$
\inf _{E_{T}} \varphi \leq 0 \quad \text { for all } x \in V \text { with }\|x\| \leq \rho .
$$

Thus condition $\left(I_{2}\right)$ of Lemma 3.4 is verified. Moreover, we have

$$
\inf _{E_{T}} \varphi(x) \leq 0
$$

Next, we consider two cases.

Case 1: $\inf _{E_{T}} \varphi<0$. Then the proof of Theorem 3.1 is finished directly due to Lemma 3.4.

Case 2: $\inf _{E_{T}} \varphi \geq 0$. Due to (8), we obtain

$$
\varphi(x)=\inf _{E_{T}} \varphi=0 \quad \text { for all } x \in E_{T} .
$$


This means that all $x \in V$ with $\|x\| \leq \rho$ are solutions of equation (1). Hence the proof of Theorem 3.1 is finished.

\section{The proof of Corollary 3.2 is omitted since it is similar to that of Theorem 3.1.}

\section{Acknowledgements}

The authors sincerely thank the reviewers for their valuable suggestions and useful comments that have led to the present improved version.

\section{Funding}

The work is supported by the Science Foundation of Education Department for Hubei Provincial, China (No. D20172905).

\section{Competing interests}

The authors declare that they have no competing interests.

\section{Authors' contributions}

SM chose the problem and drafted the manuscript with $\mathrm{ZHH}$. QJ conceived the solution method. All authors read and approved the final manuscript.

\section{Publisher's Note}

Springer Nature remains neutral with regard to jurisdictional claims in published maps and institutional affiliations.

Received: 14 November 2017 Accepted: 17 July 2018 Published online: 02 August 2018

\section{References}

1. Agarwal, R.P.: Difference Equations and Inequalities. Theory, Methods, and Applications, 2nd edn. Monographs and Textbooks in Pure and Applied Mathematics, vol. 228. Dekker, New York (2000)

2. Agarwal, R.P., Perera, K., O'Regan, D.: Multiple positive solutions of singular and nonsingular discrete problems via variational methods. Nonlinear Anal. 58, 69-73 (2004)

3. Agarwal, R.P., Perera, K., O’Regan, D.: Multiple positive solutions of singular discrete $p$-Laplacian problems via variational methods. Adv. Differ. Equ. 2005, 93-99 (2005)

4. Brezis, H., Nirenberg, L.: Remarks on finding critical points. Commun. Pure Appl. Math. 44, 939-963 (1991)

5. Cabada, A., lannizzotto, A., Tersian, S.: Multiple solutions for discrete boundary value problems. J. Math. Anal. Appl. $356,418-428(2009)$

6. Cai, X.C., Yu, J.S.: Existence theorems of periodic solutions for second-order nonlinear difference equations. Adv. Differ. Equ. 2008, Article ID 247071 (2008)

7. Chen, S.J., Tang, C.L.: Multiple periodic solutions for superquadratic first-order discrete Hamiltonian systems. Appl. Math. Comput. 208, 495-500 (2009)

8. Chen, S.Z.: Disconjugacy, disfocality, and oscillation of second order difference equation. J. Differ. Equ. 107, 383-394 (1994)

9. D'Agui, G., Mawhin, J., Sciammett, A.: Positive solutions for a discrete two point nonlinear boundary value problem with p-Laplacian. J. Math. Anal. Appl. 447, 383-397 (2017)

10. Guo, Z.M., Yu, J.S.: The existence of periodic and subharmonic solutions of subquadratic second order difference equations. J. Lond. Math. Soc. (2) 68(2), 419-430 (2003)

11. Guo, Z.M., Yu, J.S.: Existence of periodic and subharmonic solutions for second-order superlinear difference equations. Sci. China Ser. A 46(4), 506-515 (2003)

12. Jiang, D., Chu, J., O'Regan, D., Agarwal, R.P.: Positive solutions for continuous and discrete boundary value problems to the one-dimension p-Laplacian. Math. Inequal. Appl. 7, 523-534 (2004)

13. Jiang, Q., Ma, S., Hu, Z.H.: Existence of multiple periodic solutions for second-order discrete Hamiltonian systems with partially periodic potentials. Electron. J. Differ. Equ. 2016, Article ID 307 (2016)

14. Luo, Z.M., Zhang, X.Y.: Existence of nonconstant periodic solutions for a nonlinear discrete system involving the p-Laplacian. Bull. Malays. Math. Sci. Soc. (2) 35(2), 373-382 (2012)

15. Pankov, A., Zakharchenko, N.: On some discrete variational problems. Acta Appl. Math. 65, 295-303 (2005)

16. Pankov, A., Zakharchenko, N.: Solutions in discrete nonlinear Schrödinger equation with saturable non-linearity. Proc. R. Soc. Lond. Ser. A 464, 3219-3236 (2008)

17. Peil, T., Peterson, A.: Criteria for C-disfocality of a self-adjoint vector difference equation. J. Math. Anal. Appl. 179, 512-524 (1993)

18. Ren, Z., Li, J., Shi, H.: Existence of periodic solutions for second-order nonlinear difference equations. J. Nonlinear Sci. Appl. 9, 1505-1514 (2016)

19. Yu, J.S., Guo, Z.M., Zou, X.F.: Periodic solutions of second order self-adjoint difference equations. J. Lond. Math. Soc. 71, $146-160$ (2005)

20. Zhang, R.Y., Wang, Z.C., Yu, J.S.: Necessary and sufficient conditions for the existence of positive solutions of nonlinear difference equations. Fields Inst. Commun. 42, 385-396 (2004) 